\title{
EXPONENTIAL MEAN-SQUARE STABILITY OF NUMERICAL SOLUTIONS TO STOCHASTIC DIFFERENTIAL EQUATIONS
}

\author{
DESMOND J. HIGHAM, XUERONG MAO AND ANDREW M. STUART
}

\begin{abstract}
Positive results are proved here about the ability of numerical simulations to reproduce the exponential mean-square stability of stochastic differential equations (SDEs). The first set of results applies under finite-time convergence conditions on the numerical method. Under these conditions, the exponential mean-square stability of the SDE and that of the method (for sufficiently small step sizes) are shown to be equivalent, and the corresponding second-moment Lyapunov exponent bounds can be taken to be arbitrarily close. The required finite-time convergence conditions hold for the class of stochastic theta methods on globally Lipschitz problems. It is then shown that exponential mean-square stability for non-globally Lipschitz SDEs is not inherited, in general, by numerical methods. However, for a class of SDEs that satisfy a one-sided Lipschitz condition, positive results are obtained for two implicit methods. These results highlight the fact that for long-time simulation on nonlinear SDEs, the choice of numerical method can be crucial.
\end{abstract}

\section{Introduction}

Suppose that we are required to find out whether a stochastic differential equation (SDE) is exponentially stable in mean square. In the absence of an appropriate Lyapunov function, we may carry out careful numerical simulations using a numerical method with a 'small' step size $\Delta t$. Two key questions then follow.

(Q1) If the SDE is exponentially stable in mean square, will the numerical method be exponentially stable in mean square for sufficiently small $\Delta t$ ?

(Q2) If the numerical method is exponentially stable in mean square for small $\Delta t$, can we infer that the underlying SDE is exponentially stable in mean square?

These questions deal with an asymptotic $(t \rightarrow \infty)$ property, and hence they cannot be answered directly by applying traditional finite-time convergence results.

Results that answer $(\mathrm{Q} 1)$ and $(\mathrm{Q} 2)$ for scalar, linear systems can be found in $[\mathbf{6 ,} \mathbf{1 4}$, 15]. Baker and Buckwar [2] consider $p$ th mean stability of numerical methods for scalar constant delay SDEs under assumptions of global Lipschitz coefficients and the existence of a Lyapunov function. Schurz [15] also has results for nonlinear SDEs, which we mention further in Section 4.

The first author was supported by a Research Fellowship from the Leverhulme Trust. The third author was supported by the Engineering and Physical Sciences Research Council of the UK under grant GR/N00340.

Received 6 June 2003, revised 1 September 2003; published 28 November 2003.

2000 Mathematics Subject Classification 65C30, 60H35

(C) 2003, Desmond J. Higham, Xuerong Mao and Andrew M. Stuart 
In Section 2.1 we give our definitions of exponential stability in mean square for the SDE and numerical method. We then introduce in Section 2.2 a natural finite-time strong convergence condition, Condition 2.3, for the numerical method. Under this condition, Theorem 2.6 shows the equivalence, for sufficiently small step sizes, of the mean-square stability of the SDE and that of the method. In Section 2.3 we strengthen the finite-time convergence condition, and we show that a similar result, Theorem 2.10 , can then be proved more simply. In Section 3 we prove related results, Theorems 3.3 and 3.4, for second-moment Lyapunov exponent bounds. The important feature of all these results is that they transfer the asymptotic question into a verification of a finite-time convergence condition. In Appendix A we show that the required finite-time convergence condition from Section 2.2 holds for the stochastic theta method on globally Lipschitz SDEs. A similar approach could be used to establish the stronger finite-time convergence condition required in Section 2.3 - in this case, the range of 'sufficiently small' step sizes for which Theorem 2.10 holds would be smaller than that for Theorem 2.6. Section 4 begins with a counterexample to illustrate that the Euler-Maruyama method does not, in general, preserve exponential mean-square stability for nonlinear SDEs that do not have a globally Lipschitz drift. We then show in Theorem 4.4 and Corollary 4.5 that positive results can be obtained for implicit methods under a one-sided Lipschitz condition on the drift.

\section{Exponential stability}

\subsection{Definitions}

Throughout this paper, let $\left(\Omega, \mathcal{F},\left\{\mathcal{F}_{t}\right\}_{t} \geqslant 0, \mathbb{P}\right)$ be a complete probability space with a filtration $\left\{\mathcal{F}_{t}\right\}_{t} \geqslant 0$ satisfying the usual conditions (that is, it is right continuous and $\mathscr{F}_{0}$ contains all $\mathbb{P}$-null sets). Let $w(t)=\left(w_{1}(t), \ldots, w_{m}(t)\right)^{T}$ be an $m$-dimensional Brownian motion defined on the probability space. Let $|\cdot|$ denote both the Euclidean norm in $\mathbb{R}^{n}$ and the trace (or Frobenius) norm in $\mathbb{R}^{n \times m}$. Also, let $L_{\mathcal{F}_{t}}^{2}\left(\Omega ; \mathbb{R}^{n}\right)$ denote the family of all $\widetilde{F}_{t^{-}}$ measurable random variables $\xi: \Omega \longrightarrow \mathbb{R}^{n}$ such that $\mathbb{E}|\xi|^{2}<\infty$.

Consider an $n$-dimensional Itô SDE,

$$
d y(t)=f(y(t)) d t+g(y(t)) d w(t)
$$

on $t \geqslant 0$, with initial data $y(0)=\xi \in L_{\widetilde{F}_{0}}^{2}\left(\Omega ; \mathbb{R}^{n}\right)$. We suppose that a numerical method is available which, given a step size $\Delta t>0$, computes discrete approximations $x_{k} \approx y(k \Delta t)$, with $x_{0}=\xi$. We also suppose that there is a well-defined interpolation process that extends the discrete approximation $\left\{x_{k}\right\}_{k \in \mathbb{Z}^{+}}$to a continuous-time approximation $\{x(t)\}_{t \in \mathbb{R}^{+}}$, with $x(k \Delta t)=x_{k}$. Such a process is illustrated for the class of stochastic theta methods in Appendix A.

We always assume that $f: \mathbb{R}^{n} \longrightarrow \mathbb{R}^{n}$ and $g: \mathbb{R}^{n} \longrightarrow \mathbb{R}^{n \times m}$ are such that the SDE (1) has a unique solution for any initial data $y(0)=\xi \in L_{\mathcal{F}_{0}}^{2}\left(\Omega ; \mathbb{R}^{n}\right)$, and for all $t \geqslant 0$. For detailed conditions on the existence and uniqueness of SDE solutions, we refer the reader to $[1,12]$. In this section we consider the exponential stability in mean square of the origin, which we define as follows (see $[4,9,10,11])$. We frame our definitions in terms of the stability properties of the SDE and the numerical method, rather than the zero solution, as this allows for possible perturbation of the zero solution under discretization.

DEFINITION 2.1. The SDE (1) is said to be exponentially stable in mean square if there is a pair of positive constants $\lambda$ and $M$ such that, for all initial data $\xi \in L_{\widetilde{F}_{0}}^{2}\left(\Omega ; \mathbb{R}^{n}\right)$,

$$
\mathbb{E}|y(t)|^{2} \leqslant M \mathbb{E}|\xi|^{2} e^{-\lambda t}, \quad \text { for all } t \geqslant 0 .
$$


We refer to $\lambda$ as a rate constant, and to $M$ as a growth constant.

We point out that (2) forces

$$
f(0)=0 \text { and } g(0)=0
$$

in equation (1). To see this, take the initial value $\xi=0$. By (2), the solution of equation (1) must then remain zero, and so

$$
0=0+\int_{0}^{1} f(0) d s+\int_{0}^{1} g(0) d w(s)=f(0)+g(0) w(1) .
$$

Taking expectations on both sides yields $f(0)=0$. Consequently, $g(0) w(1)=0$, which implies that $g(0)=0$ too, since $w(1)$ is a normally distributed random variable. By a change of origin, other problems can be considered, but there is necessarily an $a \in \mathbb{R}^{n}$ such that $f(a)=0$ and $g(a)=0$.

Following Definition 2.1, we now define exponential stability in mean square for a numerical method that produces, through interpolation, a continuous-time approximation $x(t)$.

DEFINITION 2.2. For a given step size $\Delta t>0$, a numerical method is said to be exponentially stable in mean square on the SDE (1) if there is a pair of positive constants $\gamma$ and $N$ such that with initial data $\xi \in L_{\mathcal{F}_{0}}^{2}\left(\Omega ; \mathbb{R}^{n}\right)$,

$$
\mathbb{E}|x(t)|^{2} \leqslant N \mathbb{E}|\xi|^{2} e^{-\gamma t}, \quad \text { for all } t \geqslant 0 .
$$

\subsection{Assumption and results}

We wish to know whether the numerical method shares exponential mean-square stability with the SDE. Theorem 2.6 below resolves the issue positively for numerical methods that satisfy the following natural finite-time convergence condition.

Condition 2.3. For all sufficiently small $\Delta t$, the numerical method applied to (1) with initial condition $x_{0}=y(0)=\xi$ satisfies, for any $T>0$,

$$
\sup _{0 \leqslant t \leqslant T} \mathbb{E}|x(t)|^{2}<B_{\xi, T}
$$

where $B_{\xi, T}$ depends on $\xi$ and $T$, but not upon $\Delta t$, and

$$
\sup _{0 \leqslant t \leqslant T} \mathbb{E}|x(t)-y(t)|^{2} \leqslant\left(\sup _{0 \leqslant t \leqslant T} \mathbb{E}|x(t)|^{2}\right) C_{T} \Delta t,
$$

where $C_{T}$ depends on $T$ but not on $\xi$ and $\Delta t$.

Our notation emphasizes the dependence of $C$ upon $T$, as this is important in the subsequent analysis.

We remark that (5) says that the method has a strong finite-time convergence order of at least $1 / 2$, with a 'squared error constant' that is linearly proportional to $\sup _{0 \leqslant t \leqslant T} \mathbb{E}|x(t)|^{2}$. In Appendix A we show that the stochastic theta method satisfies Condition 2.3 when $f$ and $g$ are globally Lipschitz.

It is useful to note that Condition 2.3 implies that the solution of equation (1) satisfies

$$
\sup _{0 \leqslant t \leqslant T} \mathbb{E}|y(t)|^{2}<\infty, \quad \forall T>0 .
$$

The following lemma gives a positive answer to question (Q1) from Section 1. 
LEMMA 2.4. Assume that the SDE (1) is exponentially stable in mean square and satisfies (2). Under Condition 2.3 there exists a $\Delta t^{\star}>0$ such that for every $0<\Delta t \leqslant \Delta t^{\star}$, the numerical method is exponentially stable in mean square on the SDE (1) with rate constant $\gamma=(1 / 2) \lambda$ and growth constant $N=2 M e^{(1 / 2) \lambda T}$.

Proof. Choose $T=1+(4 \log M) / \lambda$, so that

$$
M e^{-\lambda T} \leqslant e^{-(3 / 4) \lambda T}
$$

Now, for any $\alpha>0$,

$$
\mathbb{E}|x(t)|^{2} \leqslant(1+\alpha) \mathbb{E}|x(t)-y(t)|^{2}+(1+1 / \alpha) \mathbb{E}|y(t)|^{2} .
$$

Using Condition 2.3 and (2), we see that

$$
\sup _{0 \leqslant t \leqslant 2 T} \mathbb{E}|x(t)|^{2} \leqslant(1+\alpha) \sup _{0 \leqslant t \leqslant 2 T} \mathbb{E}|x(t)|^{2} C_{2 T} \Delta t+(1+1 / \alpha) M \mathbb{E}|\xi|^{2} .
$$

If we take $\Delta t$ sufficiently small, this rearranges to

$$
\sup _{0 \leqslant t \leqslant 2 T} \mathbb{E}|x(t)|^{2} \leqslant \frac{(1+1 / \alpha) M \mathbb{E}|\xi|^{2}}{1-(1+\alpha) C_{2 T} \Delta t} .
$$

Now, taking the supremum over [T, 2T] in (8), using Condition 2.3 and the bound (9), and also the stability condition (2), gives

$$
\sup _{T \leqslant t \leqslant 2 T} \mathbb{E}|x(t)|^{2} \leqslant \frac{(1+\alpha)(1+1 / \alpha) M \mathbb{E}|\xi|^{2}}{1-(1+\alpha) C_{2 T} \Delta t} C_{2 T} \Delta t+(1+1 / \alpha) M \mathbb{E}|\xi|^{2} e^{-\lambda T}
$$

We write this as

$$
\sup _{T \leqslant t \leqslant 2 T} \mathbb{E}|x(t)|^{2} \leqslant R(\Delta t) \mathbb{E}|\xi|^{2}
$$

where

$$
R(\Delta t):=\frac{(1+\alpha)(1+1 / \alpha)}{1-(1+\alpha) C_{2 T} \Delta t} C_{2 T} \Delta t M+(1+1 / \alpha) M e^{-\lambda T} .
$$

Putting $\alpha=1 / \sqrt{\Delta t}$ and using (7), we see that for sufficiently small $\Delta t$,

$$
R(\Delta t) \leqslant 2 \sqrt{\Delta t} C_{2 T} M+(1+\sqrt{\Delta t}) e^{-(3 / 4) \lambda T} .
$$

The right-hand side of this inequality is equal to $e^{-(3 / 4) \lambda T}$ when $\Delta t=0$, and increases monotonically with $\Delta t$. Hence, by taking $\Delta t$ sufficiently small, we may ensure that

$$
R(\Delta t) \leqslant e^{-(1 / 2) \lambda T} .
$$

In (10) this gives

$$
\sup _{T \leqslant t \leqslant 2 T} \mathbb{E}|x(t)|^{2} \leqslant e^{-(1 / 2) \lambda T} \mathbb{E}|\xi|^{2}
$$

which we weaken to

$$
\sup _{T \leqslant t \leqslant 2 T} \mathbb{E}|x(t)|^{2} \leqslant e^{-(1 / 2) \lambda T} \sup _{0 \leqslant t \leqslant T} \mathbb{E}|x(t)|^{2} .
$$

Now, let $\hat{y}(t)$ be the solution to the $\operatorname{SDE}(1)$ for $t \in[T, \infty)$, with the initial condition that $\hat{y}(T)=x(T)$. Copying the previous analysis, we have

$$
\mathbb{E}|x(t)|^{2} \leqslant(1+\alpha) \mathbb{E}|x(t)-\hat{y}(t)|^{2}+(1+1 / \alpha) \mathbb{E}|\hat{y}(t)|^{2} .
$$


Taking the supremum over $[T, 3 T]$, and using the Markov property for the SDE, we may shift (2) and Condition 2.3 to $[T, 3 T]$, obtaining

$$
\sup _{T \leqslant t \leqslant 3 T} \mathbb{E}|x(t)|^{2} \leqslant(1+\alpha) \sup _{T \leqslant t \leqslant 3 T} \mathbb{E}|x(t)|^{2} C_{2 T} \Delta t+(1+1 / \alpha) M \mathbb{E}|x(T)|^{2} .
$$

This gives

$$
\sup _{T \leqslant t \leqslant 3 T} \mathbb{E}|x(t)|^{2} \leqslant \frac{(1+1 / \alpha) M \mathbb{E}|x(T)|^{2}}{1-(1+\alpha) C_{2 T} \Delta t}
$$

Now, taking the supremum over $[2 T, 3 T]$ in (12), in place of (10) we arrive at

$$
\sup _{2 T \leqslant t \leqslant 3 T} \mathbb{E}|x(t)|^{2} \leqslant R(\Delta t) \mathbb{E}|x(T)|^{2} .
$$

Continuing this approach and using (11) gives

$$
\sup _{(i+1) T \leqslant t \leqslant(i+2) T} \mathbb{E}|x(t)|^{2} \leqslant e^{-(1 / 2) \lambda T} \mathbb{E}|x(i T)|^{2}, \quad \text { for } i \geqslant 0 .
$$

From (13) we see that

$$
\begin{aligned}
\sup _{(i+1) T \leqslant t \leqslant(i+2) T} \mathbb{E}|x(t)|^{2} & \leqslant e^{-(1 / 2) \lambda T} e^{-(1 / 2) \lambda T} \sup _{(i-1) T \leqslant t \leqslant i T} \mathbb{E}|x(t)|^{2} \\
& \vdots \\
& \leqslant e^{-(1 / 2) \lambda T(i+1)} \sup _{0 \leqslant t \leqslant T} \mathbb{E}|x(t)|^{2} .
\end{aligned}
$$

Now, using $\alpha=1 / \sqrt{\Delta t}$ in (9), for sufficiently small $\Delta t$ we see that

$$
\sup _{0 \leqslant t \leqslant T} \mathbb{E}|x(t)|^{2} \leqslant 2 M \mathbb{E}|\xi|^{2} .
$$

It follows from (14) and (15) that

$$
\begin{aligned}
\sup _{(i+1) T \leqslant t \leqslant(i+2) T} \mathbb{E}|x(t)|^{2} & \leqslant e^{-(1 / 2) \lambda T(i+1)} 2 M \mathbb{E}|\xi|^{2} \\
& =2 M e^{(1 / 2) \lambda T} \mathbb{E}|\xi|^{2} e^{-(1 / 2) \lambda T(i+2)} .
\end{aligned}
$$

Hence the numerical method is exponentially stable in mean square with $\gamma=(1 / 2) \lambda$ and $N=2 M e^{(1 / 2) \lambda T}$.

The next lemma gives a positive answer to question (Q2) from Section 1.

Lemma 2.5. Assume that Condition 2.3 holds. Assume also that for a step size $\Delta t>0$, the numerical method is exponentially stable in mean square with rate constant $\gamma$ and growth constant $N$. If $\Delta t$ satisfies

$$
C_{2 T} e^{\gamma T}(\Delta t+\sqrt{\Delta t})+1+\sqrt{\Delta t} \leqslant e^{(1 / 4) \gamma T} \quad \text { and } \quad C_{T} \Delta t \leqslant 1,
$$

where $T:=1+(4 \log N) / \gamma$, then the SDE (1) is exponentially stable in mean square with rate constant $\lambda=(1 / 2) \gamma$ and growth constant $M=2 N e^{(1 / 2) \gamma T}$.

Proof. First, note that

$$
e^{-(3 / 4) \gamma T} N \leqslant e^{-(1 / 2) \gamma T}
$$

For any $\alpha>0$, we have

$$
\mathbb{E}|y(t)|^{2} \leqslant(1+\alpha) \mathbb{E}|x(t)-y(t)|^{2}+(1+1 / \alpha) \mathbb{E}|x(t)|^{2} .
$$


Using Condition 2.3 and (4) in (18), we obtain

$$
\begin{aligned}
\sup _{T \leqslant t \leqslant 2 T} \mathbb{E}|y(t)|^{2} & \leqslant(1+\alpha) \sup _{T \leqslant t \leqslant 2 T} \mathbb{E}|x(t)-y(t)|^{2}+(1+1 / \alpha) \sup _{T \leqslant t \leqslant 2 T} \mathbb{E}|x(t)|^{2} \\
& \leqslant(1+\alpha) C_{2 T} \Delta t \sup _{0 \leqslant t \leqslant 2 T} \mathbb{E}|x(t)|^{2}+(1+1 / \alpha) \sup _{T \leqslant t \leqslant 2 T} \mathbb{E}|x(t)|^{2} \\
& \leqslant(1+\alpha) C_{2 T} \Delta t N \mathbb{E}|\xi|^{2}+(1+1 / \alpha) N \mathbb{E}|\xi|^{2} e^{-\gamma T} \\
& \leqslant\left[(1+\alpha) C_{2 T} \Delta t e^{\gamma T}+(1+1 / \alpha)\right] N \mathbb{E}|\xi|^{2} e^{-\gamma T}
\end{aligned}
$$

Setting $\alpha=1 / \sqrt{\Delta t}$ gives

$$
\sup _{T \leqslant t \leqslant 2 T} \mathbb{E}|y(t)|^{2} \leqslant\left[C_{2 T} e^{\gamma T}(\Delta t+\sqrt{\Delta t})+1+\sqrt{\Delta t}\right] N \mathbb{E}|\xi|^{2} e^{-\gamma T} .
$$

Using (16) and (17), we then have

$$
\begin{aligned}
\sup _{T \leqslant t \leqslant 2 T} \mathbb{E}|y(t)|^{2} & \leqslant e^{-(3 / 4) \gamma T} N \mathbb{E}|\xi|^{2} \\
& \leqslant e^{-(1 / 2) \gamma T} \mathbb{E}|\xi|^{2} \\
& \leqslant e^{-(1 / 2) \gamma T} \sup _{0 \leqslant t \leqslant T} \mathbb{E}|y(t)|^{2} .
\end{aligned}
$$

Now let $\hat{x}(t)$ for $t \in[T, \infty)$ denote the approximation that arises from applying the numerical method with $\hat{x}(T)=y(T)$. Then, using similar arguments to those that produced (19) and (20), we have

$$
\begin{aligned}
\sup _{2 T \leqslant t \leqslant 3 T} \mathbb{E}|y(t)|^{2} & \leqslant(1+\alpha) \sup _{2 T \leqslant t \leqslant 3 T} \mathbb{E}|\hat{x}(t)-y(t)|^{2}+(1+1 / \alpha) \sup _{2 T \leqslant t \leqslant 3 T} \mathbb{E}|\hat{x}(t)|^{2} \\
& \leqslant(1+\alpha) C_{2 T} \Delta t \sup _{T \leqslant t \leqslant 3 T} \mathbb{E}|\hat{x}(t)|^{2}+(1+1 / \alpha) \sup _{2 T \leqslant t \leqslant 3 T} \mathbb{E}|\hat{x}(t)|^{2} \\
& \leqslant(1+\alpha) C_{2 T} \Delta t N \mathbb{E}|y(T)|^{2}+(1+1 / \alpha) N \mathbb{E}|y(T)|^{2} e^{-\gamma T} \\
& \leqslant\left[(1+\alpha) C_{2 T} \Delta t e^{\gamma T}+(1+1 / \alpha)\right] N \mathbb{E}|y(T)|^{2} e^{-\gamma T} \\
& \leqslant e^{-(3 / 4) \gamma T} N \mathbb{E}|y(T)|^{2} \\
& \leqslant e^{-(1 / 2) \gamma T} \mathbb{E}|y(T)|^{2} \\
& \leqslant e^{-(1 / 2) \gamma T} \sup _{T \leqslant t \leqslant 2 T} \mathbb{E}|y(t)|^{2} .
\end{aligned}
$$

Generally, this approach may be used to show that

$$
\sup _{i T \leqslant t \leqslant(i+1) T} \mathbb{E}|y(t)|^{2} \leqslant e^{-(1 / 2) \gamma T} \sup _{(i-1) T \leqslant t \leqslant i T} \mathbb{E}|y(t)|^{2}, \quad i \geqslant 1 .
$$

Hence

$$
\sup _{i T \leqslant t \leqslant(i+1) T} \mathbb{E}|y(t)|^{2} \leqslant e^{-(1 / 2) \gamma i T} \sup _{0 \leqslant t \leqslant T} \mathbb{E}|y(t)|^{2} .
$$

Now, using (16), we see that

$$
\begin{aligned}
\sup _{0 \leqslant t \leqslant T} \mathbb{E}|y(t)|^{2} & \leqslant \sup _{0 \leqslant t \leqslant T} \mathbb{E}|x(t)-y(t)|^{2}+\sup _{0 \leqslant t \leqslant T} \mathbb{E}|x(t)|^{2} \\
& \leqslant\left(C_{T} \Delta t+1\right) N \mathbb{E}|\xi|^{2} \\
& \leqslant 2 N \mathbb{E}|\xi|^{2} .
\end{aligned}
$$


In (22), this gives

$$
\sup _{i T \leqslant t \leqslant(i+1) T} \mathbb{E}|y(t)|^{2} \leqslant e^{-(1 / 2) \gamma(i+1) T} e^{(1 / 2) \gamma T} 2 N \mathbb{E}|\xi|^{2},
$$

which proves the required result.

Lemmas 2.4 and 2.5 lead to the following theorem.

Theorem 2.6. Suppose that a numerical method satisfies Condition 2.3. Then the SDE is exponentially stable in mean square if and only if there exists $a \Delta t>0$ such that the numerical method is exponentially stable in mean square with rate constant $\gamma$, growth constant $N$, step size $\Delta t$ and global error constant $C_{T}$ for $T:=1+(4 \log N) / \gamma$ satisfying conditions (16).

Proof. The 'if' part of the theorem follows directly from Lemma 2.5. To prove the 'only if' part, suppose that the SDE is exponentially stable in mean square with rate constant $\lambda$ and growth constant $M$. Lemma 2.4 shows that there is a $\Delta t^{\star}>0$ such that for any step size $0<\Delta t \leqslant \Delta t^{\star}$, the numerical method is exponentially stable in mean square with rate constant $\gamma=(1 / 2) \lambda$ and growth constant $N=2 M e^{(1 / 2) \lambda T}$. Noting that both of these constants are independent of $\Delta t$, it follows that we may reduce $\Delta t$ if necessary until (16) becomes satisfied.

We emphasize that Theorem 2.6 is an 'if and only if' result, which shows that, under Condition 2.3 and for sufficiently small $\Delta t$, the exponential stability of the method is equivalent to the exponential stability of the SDE. Thus it is feasible to investigate the exponential stability of the SDE from careful numerical simulations.

\subsection{Stronger assumption and results}

In this subsection, we strengthen the bound (5) in Condition 2.3 by forcing the 'squared error constant' to be linearly proportional to $\mathbb{E}|\xi|^{2}$, rather than $\sup _{0 \leqslant t \leqslant T} \mathbb{E}|x(t)|^{2}$. The motivation for this is twofold: (a) the proofs of the two key lemmas become simpler and more symmetric, and (b) the stronger bound (23) can be established for the case of the stochastic theta method on globally Lipschitz SDEs using an extension of the techniques given in Appendix A. However, the constant $C_{T}$ in (23) arising from that analysis is, in general, much larger than the $C_{T}$ in (5), and hence the restriction on $\Delta t$ in Theorem 2.6 is typically much less stringent than that in Theorem 2.10 .

Condition 2.7. For sufficiently small $\Delta t$, the numerical method applied to (1) with initial condition $x_{0}=y(0)=\xi$ satisfies, for any $T>0$,

$$
\sup _{0 \leqslant t \leqslant T} \mathbb{E}|x(t)|^{2}<B_{\xi, T}
$$

where $B_{\xi, T}$ depends on $\xi$ and $T$, but not upon $\Delta t$, and

$$
\sup _{0 \leqslant t \leqslant T} \mathbb{E}|x(t)-y(t)|^{2} \leqslant C_{T} \Delta t \mathbb{E}|\xi|^{2},
$$

where $C_{T}$ depends on $T$ but not on $\xi$ and $\Delta t$.

LEMMA 2.8. Assume that the SDE (1) is exponentially stable in mean square and satisfies (2), and that Condition 2.7 holds. Let $T:=1+(4 \log M) / \lambda$. Choose $\Delta t^{\star}>0$ such that for all $0<\Delta t \leqslant \Delta t^{\star}$,

$$
(\Delta t+\sqrt{\Delta t}) C_{T}+(\sqrt{\Delta t}+1) M \leqslant 2 M,
$$


and

$$
(\Delta t+\sqrt{\Delta t}) C_{2 T}+(\sqrt{\Delta t}+1) e^{-(3 / 4) \lambda T} \leqslant e^{-(1 / 2) \lambda T} .
$$

Then for all $0<\Delta t \leqslant \Delta t^{\star}$ the numerical method is exponentially stable in mean square with rate constant $\gamma=(1 / 2) \lambda$ and growth constant $N=2 M e^{(1 / 2) \lambda T}$.

Proof. Starting with (8), choosing $\alpha=1 / \sqrt{\Delta t}$ and using Condition 2.7 and (24), we have

$$
\begin{aligned}
\sup _{0 \leqslant t \leqslant T} \mathbb{E}|x(t)|^{2} & \leqslant\left[(\Delta t+\sqrt{\Delta t}) C_{T}+(\sqrt{\Delta t}+1) M\right] \mathbb{E}|\xi|^{2} \\
& \leqslant 2 M \mathbb{E}|\xi|^{2} .
\end{aligned}
$$

Now, let $\hat{y}^{[i]}(t)$ be the solution to the $\operatorname{SDE}(1)$ for $t \in[i T, \infty)$, with the initial condition $\hat{y}^{[i]}(i T)=x(i T)$. Then, using (8),

$$
\begin{aligned}
\sup _{(i+1) T \leqslant t \leqslant(i+2) T} \mathbb{E}|x(t)|^{2} \leqslant & (1+\alpha) \sup _{i T \leqslant t \leqslant(i+2) T} \mathbb{E}\left|x(t)-\hat{y}^{[i]}(t)\right|^{2} \\
& +\left(1+\frac{1}{\alpha}\right) \sup _{i T \leqslant t \leqslant(i+2) T} \mathbb{E}\left|\hat{y}^{[i]}(t)\right|^{2} .
\end{aligned}
$$

Choosing $\alpha=1 / \sqrt{\Delta t}$, using Condition 2.7 and (25), and noting that $M e^{-\lambda T} \leqslant e^{-(3 / 4) \lambda T}$, we find that

$$
\begin{aligned}
& \sup _{(i+1) T \leqslant t \leqslant(i+2) T} \mathbb{E}|x(t)|^{2} \\
& \leqslant(\Delta t+\sqrt{\Delta t}) C_{2 T} \mathbb{E}|x(i T)|^{2}+(\sqrt{\Delta t}+1) M e^{-\lambda T} \mathbb{E}|x(i T)|^{2} \\
& \leqslant\left[(\Delta t+\sqrt{\Delta t}) C_{2 T}+(\sqrt{\Delta t}+1) e^{-(3 / 4) \lambda T}\right] \sup _{i T \leqslant t \leqslant(i+1) T} \mathbb{E}|x(t)|^{2} \\
& \leqslant e^{-(1 / 2) \lambda T} \sup _{i T \leqslant t \leqslant(i+1) T} \mathbb{E}|x(t)|^{2} .
\end{aligned}
$$

Combining (26) and (28), we deduce that

$$
\begin{aligned}
\sup _{n T \leqslant t \leqslant(n+1) T} \mathbb{E}|x(t)|^{2} & \leqslant e^{-(1 / 2) \lambda n T} \sup _{0 \leqslant t \leqslant T} \mathbb{E}|x(t)|^{2} \\
& \leqslant 2 M e^{(1 / 2) \lambda T} e^{-(1 / 2) \lambda(n+1) T} \mathbb{E}|\xi|^{2},
\end{aligned}
$$

and the result follows.

The following lemma is proved by techniques almost identical to those used in the preceeding one.

Lemma 2.9. Assume that the numerical method is exponentially stable in mean square with rate constant $\gamma$ and growth constant $N$ for some step size $\Delta t>0$, and that Condition 2.3 holds. Let $T:=1+(4 \log N) / \gamma$. Then, if

$$
(\Delta t+\sqrt{\Delta t}) C_{T}+(\sqrt{\Delta t}+1) N \leqslant 2 N,
$$

and

$$
(\Delta t+\sqrt{\Delta t}) C_{2 T}+(\sqrt{\Delta t}+1) e^{-(3 / 4) \gamma T} \leqslant e^{-(1 / 2) \gamma T},
$$

then the SDE (1) is exponentially stable in mean square with rate constant $\lambda=(1 / 2) \gamma$ and growth constant $M=2 N e^{(1 / 2) \gamma T}$.

Just as Lemmas 2.4 and 2.5 combined to give Theorem 2.6, the next theorem follows from Lemmas 2.8 and 2.9 . 
THEOREm 2.10. Suppose that a numerical method satisfies Condition 2.7. Then the SDE is exponentially stable in mean square if and only if there exists a $\Delta t>0$ such that the numerical method is exponentially stable in mean square with rate constant $\gamma$, growth constant $N$, step size $\Delta t$ and global error constant $C_{T}$ for $T:=1+(4 \log N) / \gamma$ satisfying (29) and (30).

\section{Lyapunov exponents}

In Lemmas 2.4, 2.5, 2.8 and 2.9, we found new rate constants that were within a factor of $1 / 2$ of the given ones; the price we paid for this was an uncontrolled increase in the growth constants. If we are interested only in asymptotic decay rates, then it is useful to adopt the following alternative definitions, which eliminate the growth constant completely.

DEFINITION 3.1. Equation (1) is said to have second-moment Lyapunov exponent bounded by $-\lambda<0$ if, with initial data $\xi \in L_{\mathcal{F}_{0}}^{2}\left(\Omega ; \mathbb{R}^{n}\right)$,

$$
\limsup _{t \rightarrow \infty} \frac{1}{t} \log \left(\mathbb{E}|y(t)|^{2}\right) \leqslant-\lambda \text {. }
$$

Definition 3.2. For a given step size $\Delta t>0$, a numerical method is said to have secondmoment Lyapunov exponent bounded by $-\gamma<0$ on the SDE (1) if, with initial data $\xi \in L_{\mathcal{F}_{0}}^{2}\left(\Omega ; \mathbb{R}^{n}\right)$,

$$
\limsup _{t \rightarrow \infty} \frac{1}{t} \log \left(\mathbb{E}|x(t)|^{2}\right) \leqslant-\gamma
$$

We note that the $\lambda$ appearing as a rate constant in Definition 2.1 is equivalent to the $\lambda$ appearing in the second-moment Lyapunov exponent bound in Definition 3.1, and similarly for $\gamma$ in Definitions 2.2 and 3.2. Theorem 3.3 below shows that by taking $\Delta t$ sufficiently small, we can make the second-moment Lyapunov exponent bounds for the SDE and the numerical method arbitrarily close.

Theorem 3.3. Assume that Condition 2.3 holds. If the SDE (1) is exponentially stable in mean square with rate constant $\lambda$, then, given any $\varepsilon \in(0, \lambda)$, there exists $a \Delta t^{\star}>0$ such that for all $0<\Delta t \leqslant \Delta t^{\star}$ the numerical method has second-moment Lyapunov exponent bounded by $-\lambda+\varepsilon$. Conversely, if the numerical method is exponentially stable in mean square for sufficiently small step size $\Delta t$ with fixed values of $\gamma$ and $N$, then the SDE has second-moment Lyapunov exponent bounded by $-\gamma$.

Proof. This proof is similar to the proofs of Lemmas 2.4 and 2.5. Suppose that the SDE (1) has rate constant $\lambda$ and growth constant $M$. Given $\varepsilon$, choose $T=1+(2 \log M) / \varepsilon$, so that

$$
M e^{-\lambda T} \leqslant e^{-(\lambda-(1 / 2) \varepsilon) T} .
$$

Now, as in the proof of Lemma 2.4, the inequality (10) holds. (Note that $T$, and hence also the constant $C_{2 T}$, depend upon $\varepsilon$.) Using (33), we have, for sufficiently small $\Delta t$,

$$
R(\Delta t) \leqslant 2 \sqrt{\Delta t} C_{2 T} M+(1+\sqrt{\Delta t}) e^{-(\lambda-(1 / 2) \varepsilon) T},
$$

and hence there exists a $\Delta t^{\star}$ such that for all $\Delta t \leqslant \Delta t^{\star}$,

$$
\begin{aligned}
\sup _{T \leqslant t \leqslant 2 T} \mathbb{E}|x(t)|^{2} & \leqslant e^{-(\lambda-\varepsilon) T} \mathbb{E}|\xi|^{2} \\
& \leqslant e^{-(\lambda-\varepsilon) T} \sup _{0 \leqslant t \leqslant T} \mathbb{E}|x(t)|^{2} .
\end{aligned}
$$


Continuing as in the proof of Lemma 2.4, we find that for each $\Delta t \leqslant \Delta t^{\star}$ the numerical method is exponentially stable in mean square with $\gamma=\lambda-\varepsilon$ and $N=2 M e^{(\lambda-\varepsilon) T}$. The first part of the theorem then follows. (Note, however, that $T$ depends upon $\varepsilon$, and hence we cannot conclude that $N=N(\varepsilon)$ is uniformly bounded.)

To prove the converse, for any $\varepsilon \in(0, \gamma)$, we may choose $T=1+(2 \log N) / \varepsilon$ so that

$$
N e^{-\gamma T} \leqslant e^{-(\gamma-(1 / 2) \varepsilon) T},
$$

and then in place of (21) we have

$$
\sup _{T \leqslant t \leqslant 2 T} \mathbb{E}|y(t)|^{2} \leqslant e^{-(\gamma-\varepsilon) T} \sup _{0 \leqslant t \leqslant T} \mathbb{E}|y(t)|^{2} .
$$

Continuing in this way, we find that the SDE is exponentially stable in mean square with $\lambda=\gamma-\varepsilon$ and $M=2 N e^{(\gamma-\varepsilon) T}$. This means that the SDE has second-moment Lyapunov exponent bounded by $-\gamma+\varepsilon$. Since the $\varepsilon$ is arbitrary, the result then follows. (Note that, as for the first part of the proof, $T$ depends upon $\varepsilon$, and hence we cannot conclude that $M=M(\varepsilon)$ is uniformly bounded.)

In the proof of Theorem 3.3 we found it necessary to have $T$ increasing with $\varepsilon$ in order to control the intermediate growth allowed by a growth factor greater than unity. In the special case where the growth factor equals unity, we have the following stronger result.

Theorem 3.4. Assume that Condition 2.3 holds. If the SDE (1) is exponentially stable in mean square with rate constant $\lambda$ and growth constant $M=1$, then, given any $\varepsilon \in(0, \lambda)$, there exists a $\Delta t^{\star}>0$ such that for all $0<\Delta t \leqslant \Delta t^{\star}$ the numerical method is exponentially stable in mean square with rate constant $-\lambda+\varepsilon$ and growth constant $2 e^{\lambda}$. Conversely, if the numerical method is exponentially stable in mean square for sufficiently small step size $\Delta t$ with fixed values of $\gamma$ and $N=1$, then the $S D E$ is exponentially stable in mean square with rate constant $-\gamma$ and growth constant $2 e^{\gamma}$.

Proof. The result can be proved in a similar manner to Theorem 3.3, using $T=1$.

\section{Non-globally Lipschitz results}

The lemma below shows that the theorems of the previous two sections do not extend, in general, to the case where $f$ and $g$ are not globally Lipschitz. We note that a similar result, using the same function $f$ and a different $g$, has been derived in the context of ergodicity $[8,13,18]$.

Lemma 4.1. For the SDE (1) with $m=1, n=1, f(x)=-x-x^{3}$ and $g(x)=x$, we have

$$
\mathbb{E} y(t)^{2} \leqslant \mathbb{E} \xi^{2} e^{-t}, \quad t \geqslant 0 .
$$

Consider the Euler-Maruyama method applied to this problem, for any $0<\Delta t \leqslant 2$. Assume that $\mathbb{E} x_{k}^{6}<\infty$ for all $k \geqslant 0$. If

$$
\left(\mathbb{E} \xi^{2}\right)^{2} \geqslant \frac{6}{\Delta t^{2}}
$$

then $\mathbb{E} x_{k}^{2} \geqslant 2^{k} \mathbb{E} \xi^{2}$, and hence $\lim _{k \rightarrow \infty} \mathbb{E} x_{k}^{2}=\infty$.

Proof. The inequality (34) follows from Theorem 4.2 below, because conditions (36)-(38) hold with $\mu=1$ and $c=1$. 
The Euler-Maruyama method applied to the SDE gives

$$
x_{k+1}=x_{k}-\left(x_{k}+x_{k}^{3}\right) \Delta t+x_{k} \Delta w_{k},
$$

where $\Delta w_{k}:=w((k+1) \Delta t)-w(k \Delta t)$. It follows that

$$
x_{k+1}^{2}=(1-\Delta t)^{2} x_{k}^{2}-2 x_{k}^{4} \Delta t(1-\Delta t)+\Delta t^{2} x_{k}^{6}+p\left(x_{k}, \Delta t\right) \Delta w_{k}+\Delta w_{k}^{2} x_{k}^{2} .
$$

Here, $p\left(x_{k}, \Delta t\right)$ is a polynomial in $x_{k}$ and $\Delta t$, whose precise form is not relevant to our analysis. So, using the bound

$$
2 x^{2} \Delta t(1-\Delta t) \leqslant \delta(1-\Delta t)^{2}+\delta^{-1} \Delta t^{2} x^{4}, \quad \text { with } \delta=2,
$$

along with $\mathbb{E} X^{6} \geqslant\left(\mathbb{E} X^{2}\right)^{3}$, we have

$$
\begin{aligned}
\mathbb{E} x_{k+1}^{2} & \geqslant-(1-\Delta t)^{2} \mathbb{E} x_{k}^{2}+(1 / 2) \Delta t^{2} \mathbb{E} x_{k}^{6} \\
& \geqslant \mathbb{E} x_{k}^{2}\left[(1 / 2) \Delta t^{2}\left(\mathbb{E} x_{k}^{2}\right)^{2}-1\right] .
\end{aligned}
$$

If (35) holds, then, by induction, $\left(\mathbb{E} x_{k}^{2}\right)^{2} \geqslant 6 /\left(\Delta t^{2}\right)$ and $\mathbb{E} x_{k}^{2} \geqslant 2^{k} \mathbb{E} \xi^{2}$ for all $k$.

This example rules out the possibility of extending the results in Sections 2 and 3 to general nonlinear SDEs. It is therefore reasonable to seek results for specific problem classes and specific numerical methods, an approach that we briefly pursue here.

It is appropriate at this stage to mention the work of Schurz [15, Chapter 8]. Although Schurz does not address questions (Q1) and (Q2) of Section 1 directly, he has results in a similar spirit. Under conditions that include (36)-(38) below, Schurz proves a result about the propagation of initial perturbations for the backward Euler method (39); see [15, Theorem 8.3.4]. Also, under a condition that in the terminology of [17, p. 181] could be called dissipativity, Schurz proves a result about the exponential mean-square stability of the backward Euler method of the same type as Corollary 4.5 below; see [15, Corollary 8.5.2].

The structure that we impose on the SDE (1) is that there exist constants $\mu, c>0$, with $2 \mu>c$, such that the functions $f: \mathbb{R}^{n} \longrightarrow \mathbb{R}^{n}$ and $g: \mathbb{R}^{n} \longrightarrow \mathbb{R}^{n \times m}$ satisfy

$$
\begin{gathered}
\langle u-v, f(u)-f(v)\rangle \leqslant-\mu|u-v|^{2}, \\
|g(u)-g(v)|^{2} \leqslant c|u-v|^{2},
\end{gathered}
$$

for all $u, v \in \mathbb{R}^{n}$, and

$$
f(0)=g(0)=0 .
$$

The inequality (36), which is sometimes referred to as a one-sided Lipschitz condition, plays a useful role in the stability analysis of nonlinear ordinary differential equations $[3,17]$. It is, of course, intimately connected with the Lyapunov function $V(x)=|x|^{2}$. Conditions (36) and (37) are also used in [7], where finite-time strong convergence for non-locally-Lipschitz SDEs is studied. We have the following stability result.

THEOREM 4.2. Under conditions (36)-(38), any two solutions to (1) satisfy

$$
\mathbb{E}|x(t)-y(t)|^{2} \leqslant \mathbb{E}|x(0)-y(0)|^{2} e^{-(2 \mu-c) t}
$$

and the SDE is exponentially stable in mean square with rate constant $\lambda=2 \mu-c$ and growth constant $M=1$.

Proof. Application of the Itô lemma to $(1 / 2)|x-y|^{2}$ shows that

$$
(1 / 2) d|x-y|^{2} \leqslant\langle f(x)-f(y), x-y\rangle d t+(1 / 2)|g(x)-g(y)|^{2} d t+d M(x, t),
$$


where $M(x, t)$ is a martingale. Under (36)-(38), integrating and taking expectations gives the stated inequality. Since $y(t) \equiv 0$ is a solution, the exponential stability follows immediately.

We now consider the following two discrete numerical methods for (1).

- The backward Euler method:

$$
x_{k+1}=x_{k}+f\left(x_{k+1}\right) \Delta t+g\left(x_{k}\right) \Delta w_{k} .
$$

- The split-step backward Euler method:

$$
\begin{gathered}
x_{k}^{\star}=x_{k}+f\left(x_{k}^{\star}\right) \Delta t, \\
x_{k+1}=x_{k}^{\star}+g\left(x_{k}^{\star}\right) \Delta w_{k} .
\end{gathered}
$$

The backward Euler method is identical to the stochastic theta method (47) with $\theta=1$. The split-step backward Euler method is a variant that is more amenable to analysis in some cases. The results in $[6, \mathbf{1 4}, \mathbf{1 5}]$ show that the backward Euler method has good linear mean-square stability properties, and in [8] it is shown that both methods can be effective at reproducing ergodicity. Hence, these two methods are good candidates for analysis with respect to exponential mean-square stability.

The following lemma is part of [7, Lemma 3.4].

Lemma 4.3. Under conditions (36)-(38), given $b^{1}, b^{2} \in \mathbb{R}^{n}$ and $h>0$, let $a^{1}, a^{2} \in \mathbb{R}^{n}$ satisfy the implicit equations

$$
a^{(i)}-h f\left(a^{(i)}\right)=b^{(i)}, \quad i=1,2 .
$$

Then $a^{(1)}$ and $a^{(2)}$ exist, are unique, and satisfy

$$
(1+2 h \mu)\left|a^{(1)}-a^{(2)}\right|^{2} \leqslant\left|b^{(1)}-b^{(2)}\right|^{2} .
$$

THEOREM 4.4. Under conditions (36)-(38), both the backward Euler method and the splitstep backward Euler method produce a unique solution with probability 1, any two solutions satisfy

$$
\mathbb{E}\left|x_{k+1}-y_{k+1}\right|^{2} \leqslant \frac{(1+c \Delta t)}{(1+2 \mu \Delta t)} \mathbb{E}\left|x_{k}-y_{k}\right|^{2},
$$

and any solution satisfies

$$
\mathbb{E}\left|x_{k+1}\right|^{2} \leqslant \frac{(1+c \Delta t)}{(1+2 \mu \Delta t)} \mathbb{E}\left|x_{k}\right|^{2} .
$$

Proof. Existence and uniqueness follow fom Lemma 4.3. For the backward Euler method (39), Lemma 4.3 gives

$$
(1+2 \Delta t \mu)\left|x_{k+1}-y_{k+1}\right|^{2} \leqslant\left|\left[x_{k}-y_{k}\right]+\left[g\left(x_{k}\right)-g\left(y_{k}\right)\right] \Delta w_{k}\right|^{2} .
$$

Thus

$$
\begin{aligned}
(1+2 \Delta t \mu)\left|x_{k+1}-y_{k+1}\right|^{2} \leqslant\left|x_{k}-y_{k}\right|^{2} & +\left|\left[g\left(x_{k}\right)-g\left(y_{k}\right)\right] \Delta w_{k}\right|^{2} \\
& +2\left\langle x_{k}-y_{k},\left[g\left(x_{k}\right)-g\left(y_{k}\right)\right] \Delta w_{k}\right\rangle .
\end{aligned}
$$

Thus, taking conditional expectations and using the fact that

$$
\mathbb{E}|\Sigma \Delta w|^{2}=|\Sigma|_{F}^{2} \Delta t
$$

for any $\Sigma \in \mathbb{R}^{n \times m}$ and any $\Delta w \in \mathbb{R}^{n}$ with independent identically distributed $\mathcal{N}(0, \Delta t)$ 
entries, we find that

$$
\begin{aligned}
(1+2 \Delta t \mu) \mathbb{E}\left\{\left|x_{k+1}-y_{k+1}\right|^{2} \mid \mathcal{F}_{k}\right\} & \leqslant\left|x_{k}-y_{k}\right|^{2}+\left|g\left(x_{k}\right)-g\left(y_{k}\right)\right|^{2} \Delta t \\
& \leqslant(1+c \Delta t)\left|x_{k}-y_{k}\right|^{2} .
\end{aligned}
$$

(Here $\mathcal{F}_{k}$ denotes the $\sigma$-algebra of events up to and including time $t_{k}$.) Taking expectations again yields the required contractivity result (42). A similar analysis gives (42) for the split-step backward Euler method.

The inequality (43) follows because $y_{k} \equiv 0$ is a solution.

Comparing Theorems 4.2 and 4.4, we see that the two backward Euler methods successfully capture the exponential mean-square stability of the SDE. Unlike the theorems in the previous two sections, Theorem 4.4 applies for all $\Delta t>0$; this is because we are able to exploit the particular structure of the methods, rather than appealing to general asymptotic finite-time accuracy. We also note that as $\Delta t \rightarrow 0$, the decay rate approaches that for the SDE. This is formalised in the following corollary.

COROLlaRY 4.5. Under conditions (36)-(38), given any $\Delta t>0$, the backward Euler and split-step backward Euler solutions satisfy

where

$$
\mathbb{E}\left|x_{k}\right|^{2} \leqslant \mathbb{E}\left|x_{0}\right|^{2} e^{-\hat{\gamma}(\Delta t) k \Delta t}, \quad k \geqslant 0,
$$

$$
\hat{\gamma}(\Delta t):=\frac{1}{\Delta t} \log \left[\frac{1+2 \mu \Delta t}{1+c \Delta t}\right]>0 .
$$

Also, given any $\varepsilon>0$, there exists a $\Delta t^{\star}>0$ such that for all $0<\Delta t \leqslant \Delta t^{\star}$,

$$
\mathbb{E}\left|x_{k}\right|^{2} \leqslant \mathbb{E}\left|x_{0}\right|^{2} e^{(-(2 \mu-c)+\varepsilon) k \Delta t}, \quad \text { for all } k \geqslant 0 \text {. }
$$

Proof. The inequality (45) follows directly from Theorem 4.4, and (46) is then a consequence of the fact that $\hat{\gamma}(\Delta t)=2 \mu-c+O(\Delta t)$.

\section{Appendix A. Condition 2.3 for the stochastic theta method}

We focus here on the class of stochastic theta methods, defined by

$$
x_{k+1}=x_{k}+(1-\theta) f\left(x_{k}\right) \Delta t+\theta f\left(x_{k+1}\right) \Delta t+g\left(x_{k}\right)[w((k+1) \Delta t)-w(k \Delta t)],
$$

where $\theta \in[0,1]$ is a free parameter that is specified a priori. Generally, (47) represents a nonlinear system that is to be solved for $x_{k+1}$. With the choice $\theta=0$, definition (47) is the widely-used Euler-Maruyama method. In this case, (47) is an explicit equation that defines $x_{k+1}$. We introduce the continuous approximation

where

$$
x(t)=\xi+\int_{0}^{t}(1-\theta) f\left(z_{1}(s)\right)+\theta f\left(z_{2}(s)\right) d s+\int_{0}^{t} g\left(z_{1}(s)\right) d w(s),
$$

$$
z_{1}(t)=\sum_{k=0}^{\infty} x_{k} \mathbf{1}_{[k \Delta t,(k+1) \Delta t)}(t), \quad \text { and } \quad z_{2}(t)=\sum_{k=0}^{\infty} x_{k+1} \mathbf{1}_{[k \Delta t,(k+1) \Delta t)}(t),
$$

with $\mathbf{1}_{G}$ denoting the indicator function for the set $G$. It is easily shown that $x(k \Delta t)=x_{k}$, and hence $x(t)$ is an interpolant to the discrete stochastic theta method solution. We also note that $z_{1}(k \Delta t)=z_{2}((k-1) \Delta t)=x_{k}$.

It is useful to note that if the stochastic theta method is exponentially stable in mean square for some $\Delta t$, then (3) holds. To show this, following the arguments in Section 1 , 
we insert $\xi=0$ in (47) to give $0=f(0) \Delta t+g(0) w(\Delta t)$. Taking expected values gives $f(0)=0$, and since $w(\Delta t)$ is normally distributed, $g(0) w(\Delta t)=0$ implies that $g(0)=0$. In all the results of Sections 2 and 3, we assume that either the SDE or the numerical method is exponentially stable in mean square; it follows that we will always have (3).

We impose a global Lipschitz condition on the coefficients of the SDE (1); that is, $|f(x)-f(y)|^{2} \leqslant K_{1}|x-y|^{2}$ and $|g(x)-g(y)|^{2} \leqslant K_{2}|x-y|^{2}, \quad$ for all $x, y \in \mathbb{R}^{n}$,

where $K_{1}$ and $K_{2}$ are constants. We also note that (49) and (3) combine to give the linear growth bound

$$
|f(x)|^{2} \leqslant K_{1}|x|^{2} \quad \text { and } \quad|g(x)|^{2} \leqslant K_{2}|x|^{2}, \quad \text { for all } x \in \mathbb{R}^{n} .
$$

Our first lemma concerns the existence of solutions to the implicit equation (47). This is a direct analogue of the classical deterministic theory; see, for example, [5, Theorem 7.2].

Lemma A.1. Under the global Lipschitz condition (49), if $K_{1} \theta \Delta t<1$, then equation (47) can be solved uniquely for $x_{k+1}$, with probability 1 .

Proof. Writing (47) as $x_{k+1}=F\left(x_{k+1}\right)$, we have, using (49),

$$
\begin{aligned}
|F(u)-F(v)| & =|\theta f(u) \Delta t-\theta f(v) \Delta t| \\
& \leqslant K_{1} \theta \Delta t|u-v| .
\end{aligned}
$$

The result follows from the classical Banach contraction mapping theorem [16].

Lemma A.2. Under (3) and the global Lipschitz condition (49), for sufficiently small $\Delta t$, the discrete stochastic theta method solution (47) satisfies

$$
\mathbb{E}\left|x_{k+1}\right|^{2} \leqslant 2 \mathbb{E}\left|x_{k}\right|^{2}, \quad \text { for all } k \geqslant 0 .
$$

Proof. From (47) we have

$$
\begin{aligned}
\mathbb{E}\left|x_{k+1}\right|^{2} \leqslant 1.5 \mathbb{E}\left|x_{k}\right|^{2} & +6 \Delta t^{2} \mathbb{E}\left|(1-\theta) f\left(x_{k}\right)+\theta f\left(x_{k+1}\right)\right|^{2} \\
& +6 \mathbb{E}\left|g\left(x_{k}\right)[w((k+1) \Delta t)-w(k \Delta t)]\right|^{2} .
\end{aligned}
$$

Noting that

$$
\left|(1-\theta) f\left(x_{k}\right)+\theta f\left(x_{k+1}\right)\right|^{2} \leqslant\left|f\left(x_{k}\right)\right|^{2}+\left|f\left(x_{k+1}\right)\right|^{2}
$$

and using (50), we further compute that

$$
\begin{aligned}
\mathbb{E}\left|x_{k+1}\right|^{2} & \leqslant 1.5 \mathbb{E}\left|x_{k}\right|^{2}+6 \Delta t^{2} K_{1}\left(\mathbb{E}\left|x_{k}\right|^{2}+\mathbb{E}\left|x_{k+1}\right|^{2}\right)+6 K_{2} \Delta t \mathbb{E}\left|x_{k}\right|^{2} \\
& =\left(1.5+6 \Delta t^{2} K_{1}+6 K_{2} \Delta t\right) \mathbb{E}\left|x_{k}\right|^{2}+6 \Delta t^{2} K_{1} \mathbb{E}\left|x_{k+1}\right|^{2}
\end{aligned}
$$

If $\Delta t$ is sufficiently small for

$$
6 \Delta t K_{1}<1 \text { and } \frac{1.5+6 \Delta t^{2} K_{1}+6 K_{2} \Delta t}{1-6 \Delta t^{2} K_{1}} \leqslant 2
$$

we then have

$$
\mathbb{E}\left|x_{k+1}\right|^{2} \leqslant 2 \mathbb{E}\left|x_{k}\right|^{2},
$$

as required. 
Lemma A.3. Under (3) and the global Lipschitz condition (49), for sufficiently small $\Delta t$, the stochastic theta method solution (48) satisfies

$$
\sup _{0 \leqslant t \leqslant T} \mathbb{E}|x(t)|^{2}<\infty
$$

and

$$
\sup _{0 \leqslant t \leqslant T}\left\{\mathbb{E}\left|x(t)-z_{1}(t)\right|^{2} \vee \mathbb{E}\left|x(t)-z_{2}(t)\right|^{2}\right\} \leqslant\left(2 K_{2}+1\right) \Delta t \sup _{0 \leqslant t \leqslant T} \mathbb{E}|x(t)|^{2},
$$

for all $T>0$.

Proof. Given any $0 \leqslant t \leqslant T$, let $k=[T / \Delta t]$, the integer part of $T / \Delta t$, so $k \Delta t \leqslant t<$ $(k+1) \Delta t$. It follows from (48) that

$$
x(t)=x_{k}+\left[(1-\theta) f\left(x_{k}\right)+\theta f\left(x_{k+1}\right)\right](t-k \Delta t)+g\left(x_{k}\right)(w(t)-w(k \Delta t)) .
$$

Compute

$$
\mathbb{E}|x(t)|^{2} \leqslant 3\left[\mathbb{E}\left|x_{k}\right|^{2}+K_{1} \Delta t^{2}\left(\mathbb{E}\left|x_{k}\right|^{2}+\mathbb{E}\left|x_{k+1}\right|^{2}\right)+\Delta t \mathbb{E}\left|x_{k}\right|^{2}\right] .
$$

By Lemma A.2, we obtain the assertion (51). To show that (52) holds, we note that

$$
\begin{aligned}
x(t)-z_{1}(t)= & {\left[(1-\theta) f\left(x_{k}\right)+\theta f\left(x_{k+1}\right)\right](t-k \Delta t) } \\
& +g\left(x_{k}\right)[w(t)-w(k \Delta t)],
\end{aligned}
$$

and

$$
\begin{aligned}
z_{2}(t)-x(t)= & {\left[(1-\theta) f\left(x_{k}\right)+\theta f\left(x_{k+1}\right)\right]((k+1) \Delta t-t) } \\
& +g\left(x_{k}\right)[w((k+1) \Delta t)-w(t)] .
\end{aligned}
$$

By Lemma A.2, we compute from (53) that

$$
\begin{aligned}
\mathbb{E}\left|x(t)-z_{1}(t)\right|^{2} & \leqslant 2 \Delta t^{2} K_{1}\left(\mathbb{E}\left|x_{k}\right|^{2}+\mathbb{E}\left|x_{k+1}\right|^{2}\right)+2 \Delta t K_{2} \mathbb{E}\left|x_{k}\right|^{2} \\
& \leqslant 6 \Delta t^{2} K_{1} \mathbb{E}\left|x_{k}\right|^{2}+2 \Delta t K_{2} \mathbb{E}\left|x_{k}\right|^{2} \\
& \leqslant\left(2 K_{2}+1\right) \mathbb{E}\left|x_{k}\right|^{2} \\
& \leqslant\left(2 K_{2}+1\right) \sup _{0 \leqslant t \leqslant T} \mathbb{E}|x(t)|^{2}
\end{aligned}
$$

if $\Delta t \leqslant 1 /\left(6 K_{1}\right)$. Similarly, we can show the same upper bound for $\mathbb{E}\left|z_{2}(t)-x(t)\right|^{2}$, and hence the assertion (52) follows.

THEOREM A.4. Under (3) and the global Lipschitz condition (49), for sufficiently small $\Delta t$, the stochastic theta method solution (48) satisfies

$$
\sup _{0 \leqslant t \leqslant T} \mathbb{E}|x(t)-y(t)|^{2} \leqslant\left(\sup _{0 \leqslant t \leqslant T} \mathbb{E}|x(t)|^{2}\right) C_{T} \Delta t, \quad \text { for all } T>0,
$$

where

$$
C_{T}=2 T\left(2 K_{2}+1\right)\left(4 K_{1} T+K_{2}\right) e^{2 T\left(K_{1} T+K_{2}\right)},
$$

which is independent of $\Delta t$. This, together with Lemma A.3, shows that under (3) and the global Lipschitz condition (49), the stochastic theta method satisfies Condition 2.3. 
Proof. It follows from (1) and (48) that for any $0 \leqslant t \leqslant T$,

$$
\begin{aligned}
x(t)-y(t)= & \int_{0}^{t}\left((1-\theta)\left[f\left(z_{1}(s)\right)-f(y(s))\right]+\theta\left[f\left(z_{2}(s)\right)-f(y(s))\right]\right) d s \\
& +\int_{0}^{t} g\left(z_{1}(s)\right)-g(y(s)) d w(s) .
\end{aligned}
$$

Hence

$$
\begin{aligned}
& \mathbb{E}|x(t)-y(t)|^{2} \\
& \leqslant 2\left[K_{1} T \int_{0}^{t}\left[\mathbb{E}\left|z_{1}(s)-y(s)\right|^{2}+\mathbb{E}\left|z_{2}(s)-y(s)\right|^{2}\right] d s\right. \\
&\left.\quad+K_{2} \int_{0}^{t} \mathbb{E}\left|z_{1}(s)-y(s)\right|^{2} d s\right] \\
& \leqslant 2\left[\left(2 K_{1} T+K_{2}\right)\left\{\int_{0}^{t} \mathbb{E}\left|z_{1}(s)-x(s)\right|^{2} d s+\int_{0}^{t} \mathbb{E}|x(s)-y(s)|^{2} d s\right\}\right. \\
&\left.\quad+2 K_{1} T\left\{\int_{0}^{t} \mathbb{E}\left|z_{2}(s)-x(s)\right|^{2} d s+\int_{0}^{t} \mathbb{E}|x(s)-y(s)|^{2} d s\right\}\right] .
\end{aligned}
$$

Using Lemma A.3, we then have

$$
\begin{aligned}
\mathbb{E}|x(t)-y(t)|^{2} \leqslant & \left(8 K_{1} T+2 K_{2}\right) \int_{0}^{t} \mathbb{E}|x(s)-y(s)|^{2} d s \\
& +T\left(2 K_{2}+1\right)\left(8 K_{1} T+2 K_{2}\right) \Delta t\left(\sup _{0 \leqslant t \leqslant T} \mathbb{E}|x(t)|^{2}\right) .
\end{aligned}
$$

From an application of the continuous Gronwall lemma (see, for example, [12]), we obtain a bound of the form

$$
\mathbb{E}|x(t)-y(t)|^{2} \leqslant\left(\sup _{0 \leqslant t \leqslant T} \mathbb{E}|x(t)|^{2}\right) C_{T} \Delta t .
$$

Since this holds for any $t \in[0, T]$, the assertion (55) must hold.

\section{References}

1. L. ARNOLD, Stochastic differential equations: theory and applications (Wiley, New York, 1972). 298

2. C. T. H. BAKER and E. BuckWAR, 'Exponential stability in $p$-th mean of solutions, and of convergent Euler-type solutions, to stochastic delay differential equations', Numerical Analysis Report 390, University of Manchester, 2001. 297

3. K. DEKKER and J. G. VERWER, Stability of Runge-Kutta methods for stiff nonlinear equations (North-Holland, Amsterdam, 1984). 307

4. A. Friedman, Stochastic differential equations and their applications (Academic Press, New York, 1976). 298

5. E. HAIrer, S. P. NøRSETt and G. WANnER, Solving ordinary differential equations I: nonstiff problems, 2nd edn (Springer, Berlin, 1993). 310

6. D. J. Higham, 'Mean-square and asymptotic stability of the stochastic theta method', SIAM J. Numer. Anal. 38 (2000) 753-769. 297, 308 
7. D. J. Higham, X. Mao and A. M. Stuart, 'Strong convergence of numerical methods for nonlinear stochastic differential equations', SIAM J. Numer. Anal. 40 (2002) 10411063. 307, 308

8. J. Mattingly, A. M. Stuart and D. J. Higham, 'Ergodicity for SDEs and approximations: locally Lipschitz vector fields and degenerate noise', Stoch. Process. Appl. 101 (2002) 185-232. 306, 308

9. R. Z. KHASMINSKII, Stochastic stability of differential equations (Sijthoff and Noordhoff, Alphen aan den Rijn, 1981). 298

10. X. Mao, Stability of stochastic differential equations with respect to semimartingales (Longman Scientific and Technical, London, 1991). 298

11. X. MAO, Exponential stability of stochastic differential equations (Marcel Dekker, New York, 1994). 298

12. X. Maо, Stochastic differential equations and applications (Horwood, Chichester, 1997). 298, 312

13. G. O. Roberts and R. L. Tweedie, 'Exponential convergence of Langevin diffusions and their discrete approximations', Bernoulli 2 (1996) 341-363. 306

14. Y. SAito and T. Mitsui, 'Stability analysis of numerical schemes for stochastic differential equations', SIAM J. Numer. Anal. 33 (1996) 2254-2267. 297, 308

15. H. Schurz, Stability, stationarity, and boundedness of some implicit numerical methods for stochastic differential equations and applications, $\mathrm{PhD}$ Thesis, Humboldt University (Logos Verlag, Berlin, 1997). 297, 307, 308

16. D. R. Smart, Fixed point theorems (Cambridge University Press, 1974). 310

17. A. M. Stuart and A. R. Humphries, Dynamical systems and numerical analysis (Cambridge University Press, 1996). 307

18. D. TAlay, Approximation of the invariant probability measure of stochastic Hamiltonian dissipative systems with non globally Lipschitz co-efficients, Progress in Stochastic Structural Dynamics 152 (ed. R. Bouc and C. Soize, L.M.A.-CNRS, 1999). 306

Desmond J. Higham djh@maths.strath.ac.uk

http://www.maths.strath.ac.uk/ aas96106/

Department of Mathematics

University of Strathclyde

Glasgow G1 1XH

Xuerong Mao xuerongestams.strath.ac.uk

http://www.stams.strath.ac.uk/people/staff/bios/XuerongMao/index.php

Department of Statistics and Modelling Science

University of Strathclyde

Glasgow G1 1XH

Andrew M. Stuart stuart@maths.warwick.ac.uk

http://www.maths.warwick.ac.uk/staff/stuart.html

Mathematics Institute

University of Warwick

Coventry CV4 7AL 